\title{
Laser-induced modulation of the Landau level structure in single-layer graphene
}

\author{
Alexander López, ${ }^{1, *}$ Antonio Di Teodoro, ${ }^{1}$ John Schliemann, ${ }^{2}$ Bertrand Berche, ${ }^{3}$ and Benjamin Santos ${ }^{4}$ \\ ${ }^{1}$ School of Physics Yachay Tech, Yachay City of Knowledge 100119-Urcuqui, Ecuador \\ ${ }^{2}$ Institute for Theoretical Physics, University of Regensburg, D-93040 Regensburg, Germany \\ ${ }^{3}$ Statistical Physics Group, Institut Jean Lamour UMR 7198, CNRS - Université de Lorraine, \\ Campus de Nancy, B.P. 70239, F-54506 Vandouvre lès Nancy Cedex, France, EU \\ ${ }^{4}$ INRS-EMT, Université du Québec, 1650 Blvd. Lionel-Boulet, Varennes, J3X 1S2, Canada \\ (Received 29 July 2015; revised manuscript received 27 September 2015; published 7 December 2015)
}

\begin{abstract}
We present perturbative analytical results of the Landau level quasienergy spectrum, autocorrelation function, and out-of-plane pseudospin polarization for a single graphene sheet subject to intense circularly polarized Terahertz radiation. For the quasienergy spectrum, we find a striking nontrivial level-dependent dynamically induced gap structure. This photoinduced modulation of the energy band structure gives rise to shifts of the revival times in the autocorrelation function and it also leads to modulation of the oscillations in the dynamical evolution of the out-of-plane pseudospin polarization, which measures the angular momentum transfer between light and graphene electrons. For a coherent state, chosen as an initial pseudospin configuration, the dynamics induces additional quantum revivals of the wave function that manifest as shifts of the maxima and minima of the autocorrelation function, with additional partial revivals and beating patterns. These additional maxima and beating patterns stem from the effective dynamical coupling of the static eigenstates. We discuss the possible experimental detection schemes of our theoretical results and their relevance in new practical implementation of radiation fields in graphene physics.
\end{abstract}

DOI: 10.1103/PhysRevB.92.235411

PACS number(s): 78.67.Wj, 71.70.Di, 72.80.Vp

\section{INTRODUCTION}

The dynamical control of the transport properties of Dirac fermions in the condensed matter realm is currently an intense research topic. These Dirac fermions have been shown to emerge as the low energy excitations of two-dimensional systems with a honeycomb lattice structure as it occurs in graphene [1-3]. Recent theoretical [4-6] and experimental [7] works have discussed the role of radiation fields in the manipulation of the transport properties of monolayer graphene samples. By focusing on the Terahertz frequency regime, particular attention is paid to the tunability of the induced band gaps. In addition, the possibility of generating topological insulating behavior was theoretically put forward both in the static [8] and dynamical regimes [9-11].

In presence of a perpendicular quantizing magnetic field $\mathbf{B}=B \hat{z}$, the static spectrum of single layer graphene possesses a $\sqrt{B}$ field dependence which strikingly contrasts the linear $B$ dependence for conventional nonrelativistic 2DEG [1]. In addition, the $n=0$ Landau level (LL) has only one sublattice occupied at each Dirac point. Considering the LL scenario and a topological contribution given by an excitonic gap the authors of Ref. [12] predict the appearance of Rabi oscillations when the system's initial quantum state is prepared by means of a short electric pulse and the subsequent dynamics is controlled by the oscillations between the dynamically coupled LL.

In this work we theoretically analyze the dynamical manipulation of the LL structure of charge carriers on suspended monolayer graphene when a periodically driving radiation field is applied perpendicular to the sample. A similar setup was proposed in Ref. [13], where a Gaussian laser pulse is

\footnotetext{
*To whom correspondence should be addressed: alexander.lopez@physik.uni-regensburg.de
}

introduced and the resulting dynamics is analyzed. In that work, the authors discuss the dynamics of Zitterbewegung which is described in terms of the radiation field emitted by the accelerated charge carriers in graphene. In our proposal we consider a continuous laser field and thus make use of Floquet's theorem to recast the dynamics in an explicitly time-independent fashion but without need to resort to infinitedimensional Fourier-mode expansion. Our approach has the advantage of providing an analytical description of the driven evolution of relevant physical quantities as the pseudospin polarization which is a measure of the angular momentum exchange among the charge carriers and the radiation field [14]. In the following we explicitly show how our semianalytical results are relevant at high values of the quantizing magnetic field $B$, where the coupling to the radiation field leads to nontrivial qualitative modifications of the dynamical behavior of relevant physical quantities within the perturbative regime. To the best of our knowledge, our work constitutes the first approach to the photoinduced modulation of the Landau level structure in single-layer graphene in presence of an intense and continuously applied laser field. However, we would like to mention that a recent work [15] has addressed the role of intense radiation field. Yet, the authors of this work consider a quantized radiation field and do not address the Landau level structure scenario in single-layer graphene.

The paper is organized as follows. In Sec. II we present the model and summarize the perturbative results for the quasienergy spectrum. In Sec. III we study the dynamics of the autocorrelation function and pseudospin polarization for two initially prepared states. First we consider an eigenstate of the static Hamiltonian that has vanishing pseudospin polarization in the static regime. Next, we present the same analysis for an initially prepared coherent state, with static finite pseudospin polarization and highlight the main differences with respect to the other initial configuration. In Sec. IV we discuss our 
main results and in Sec. V we present concluding remarks and argue on the experimental implementation of our proposed theoretical setup. Finally, in the Appendix we summarize some mathematical calculations arising during the perturbative analysis.

\section{MODEL}

In this section we focus on the low energy properties of noninteracting spinless charge carriers in a suspended monolayer graphene subject to a perpendicular, uniform, and constant magnetic field $\mathbf{B}=B \hat{z}$. The dynamics is governed by Dirac's Hamiltonian. In coordinate representation it reads

$$
\mathcal{H}_{\eta}(\mathbf{r})=v_{F}\left(\eta \pi_{x} \sigma_{x}+\pi_{y} \sigma_{y}\right),
$$

where $v_{F} \sim 10^{6} \mathrm{~m} / \mathrm{s}$ is the Fermi velocity in graphene. In addition, the canonical momenta $\pi_{j}=p_{j}+e A_{j}(j=x, y)$ contain the vector potential $(\nabla \times \mathbf{A}=\mathbf{B}),-e$ is the electronic charge $(e>0)$, and $\eta= \pm 1$ describes the valley degree of freedom. Using the definition of the magnetic length $l_{B}^{-2}=e B / \hbar$ and the cyclotron energy $\hbar \omega_{c}=\sqrt{2} v_{F} \hbar / l_{B}$ the Hamiltonian Eq. (1) at each $K\left(K^{\prime}\right)$ Dirac point, which corresponds to $\eta=+1(\eta=-1)$, can be written in the form

$$
\begin{gathered}
H_{+1}=\hbar \omega_{c}\left(\begin{array}{cc}
0 & a \\
a^{\dagger} & 0
\end{array}\right), \\
H_{-1}=-\hbar \omega_{c}\left(\begin{array}{cc}
0 & a^{\dagger} \\
a & 0
\end{array}\right),
\end{gathered}
$$

where the annihilation and creation operators are defined by standard relations as

$$
a=\frac{l_{B}\left(\pi_{x}-i \pi_{y}\right)}{\sqrt{2}} \quad \text { and } \quad a^{\dagger}=\frac{l_{B}\left(\pi_{x}+i \pi_{y}\right)}{\sqrt{2}} .
$$

The eigenenergies of the Hamiltonian (1) are then

$$
E_{n}^{s, \eta}=s \eta \sqrt{n} \hbar \omega_{c},
$$

with $s= \pm 1$. Positive (negative) values of $s \eta$ represents the conduction (valence) band at each Dirac point. In addition, the integer quantum number $n=0,1,2 \ldots$ labels the Landau level (LL) structure of monolayer graphene. Using the eigenstates $|n\rangle$ of the operator $a^{\dagger} a$, the corresponding eigenstates $\left|\varphi_{n}^{s, \eta}\right\rangle$ read

$$
\begin{aligned}
\left|\varphi_{n}^{s,+1}\right\rangle & =\frac{1}{\sqrt{2}}\left(\begin{array}{c}
s|n-1\rangle \\
|n\rangle
\end{array}\right), \\
\left|\varphi_{n}^{s,-1}\right\rangle & =\frac{1}{\sqrt{2}}\left(\begin{array}{c}
-s|n\rangle \\
|n-1\rangle
\end{array}\right),
\end{aligned}
$$

for $n \neq 0$. The zero energy eigenstate $(n=0)$ is given in each case by

$$
\begin{aligned}
& \left|\varphi_{0}^{+1}\right\rangle=\left(\begin{array}{c}
0 \\
|0\rangle
\end{array}\right), \\
& \left|\varphi_{0}^{-1}\right\rangle=\left(\begin{array}{c}
|0\rangle \\
0
\end{array}\right) .
\end{aligned}
$$

Due to time-reversal symmetry, we have $\mathcal{T} H_{+1} \mathcal{T}=H_{-1}$. Let us now consider the effect of intense circularly polarized
Terahertz electromagnetic radiation, incident perpendicularly to the sample. We assume that the beam radiation spot is large enough compared to the lattice spacing so we can neglect any spatial variation. According to the standard light-matter interaction formulation, the dynamical effects of a monochromatic radiation field incident perpendicular to the sample can be described by means of a time-dependent vector potential

$$
\mathbf{A}(t)=\frac{\mathcal{E}}{\omega}(\cos \omega t, \delta \sin \omega t),
$$

where $\mathcal{E}$ and $\omega$ are, respectively, the amplitude and frequency of the electric field given in turn by the standard relation $\mathcal{E}(t)=-\partial_{t} \mathbf{A}(t)$. In addition, we are using $\delta=+1(\delta=-1)$ for right (left) circular polarization. We are using circular polarization because it has been shown to provide the maximal photoinduced band gap [16]. Starting from the ordinary dipolar interaction term $-e \mathbf{p} \cdot \mathbf{A}(t)$, introduced to the tight-binding Hamiltonian via the Peierls substitution, we can evaluate the effects of the driving at each Dirac point as

$$
V_{\eta}=e v_{F}\left[\eta \sigma_{x} A_{x}(t)+\delta \sigma_{y} A_{y}(t)\right],
$$

which explicitly reads

$$
V_{\eta}=\xi \eta\left(\sigma_{x} \cos \omega t+\eta \delta \sigma_{y} \sin \omega t\right),
$$

with the effective coupling constant $\xi=e v_{F} \mathcal{E} / \omega$. This makes the total Hamiltonian

$$
H_{\eta}(t)=H_{\eta}+V_{\eta}(t),
$$

periodic in time $H_{\eta}(t+T)=H_{\eta}(t)$, with $T=2 \pi / \omega$ the period of oscillation of the driving field. Therefore, if we focus on the $K$ Dirac point $(\eta=1)$, the physics at the $K^{\prime}$ Dirac point $(\eta=-1)$ can be easily found by the substitutions $\xi \rightarrow-\xi$ and $\omega \rightarrow-\omega$.

Thus, let us focus on the $K$ point physics and afterwards we can make the necessary substitutions. In order to simplify the notation we set $H_{+1}=H_{0}$ and $V_{+1}(t)=V(t)$. Hence, defining rising $\sigma_{+}$and lowering $\sigma_{-}$pseudospin operators by the standard formulas

$$
\sigma_{ \pm}=\frac{\sigma_{x} \pm i \sigma_{y}}{2}
$$

the time-dependent interaction potential can be rewritten as

$$
V(t)=\xi\left(e^{-i \delta \omega t} \sigma_{+}+e^{i \delta \omega t} \sigma_{-}\right) .
$$

Now we invoke Floquet's theorem which states that the time evolution operator of the system induced by a periodic Hamiltonian can be written in the form [17]

$$
U(t)=P(t) e^{-i H_{F} t / \hbar},
$$

with $P(t)$ a periodic unitary matrix and $H_{F}$ a time-independent dynamical generator referred to as the Floquet Hamiltonian. The eigenvalues of the Floquet Hamiltonian $H_{F}$ represent the quasienergy spectrum of the periodically driven system. Typically, in order to solve for the quasienergy spectrum, one can expand each term of the time-dependent Schrödinger equation in Fourier space and numerically solve an infinite eigenvalue problem. Instead, we will take a perturbative approach as discussed below.

Accordingly, for our problem we can find approximate solutions to the dynamics by modifying slightly the analytical 
strategy presented in Ref. [18]. Then, one finds that the excitation number operator $N_{a}$, defined as

$$
N_{a}=\left(a^{\dagger} a+\frac{1}{2}\right) \mathbb{1}+\frac{\sigma_{z}}{2},
$$

which commutes with the Hamiltonian $H_{0}$ and satisfies the eigenvalue equation

$$
N_{a}\left|\varphi_{n}^{s}\right\rangle=n\left|\varphi_{n}^{s}\right\rangle .
$$

$N_{a}$ generates a time-dependent unitary transformation $|\Psi(t)\rangle=P(t)|\Phi(t)\rangle$ given as

$$
P(t)=\exp \left(-i N_{a} \delta \omega t\right),
$$

such that the time-dependent Schrödinger equation

$$
i \hbar \partial_{t}|\Psi(t)\rangle=H(t)|\Psi(t)\rangle
$$

can be transformed with a time-independent operator $H_{F}$ governing the dynamics of the problem

$$
i \hbar \partial_{t}|\Phi(t)\rangle=H_{F}|\Phi(t)\rangle,
$$

where $H_{F}$ and $|\Phi(t)\rangle$ are the Floquet Hamiltonian and Floquet eigenstate, respectively. Doing the explicit calculation, $H_{F}$ is found to be given by

$$
H_{F}=H_{0}-N_{a} \delta \hbar \omega+\xi \sigma_{x} .
$$

In the following we focus on recent experiments in the far infrared frequency domain [7] for which $\hbar \omega \approx 10 \mathrm{meV}$ and we consider values of the electric field intensities $\mathcal{E} \sim 0.15$ $\mathrm{MV} / \mathrm{m}$. Then one gets for the coupling constant $\xi \approx 10 \mathrm{meV}$ which, for frequencies $\omega$ in the Terahertz domain leads to $\xi \approx \hbar \omega$. This value is an order of magnitude smaller than the Landau level separation $\hbar \omega_{c} \approx 116 \mathrm{meV}$, for $B=10 \mathrm{~T}$. For larger frequencies and stronger magnetic field intensities, the ratio $\xi / \hbar \omega_{c}$ tends to be smaller. Therefore, we can perform a perturbative treatment in the effective coupling parameter $\lambda=\xi / \hbar \omega_{c}<1$.

We should remark that although our radiation field is intense, it is one order of magnitude smaller than the numerical estimates used in Ref. [6] for which one gets $\mathcal{E} \sim 1.5 \mathrm{MV} / \mathrm{m}$. Yet, the experimental setup used in Ref. [7] consisted of infrared radiation field with power $P=20 \mathrm{~mW}$, sample areas equal to $A=3 \times 3 \mathrm{~mm}^{2}$ and $A=5 \times 5 \mathrm{~mm}^{2}$; thus, one gets electric field intensities of order $\mathcal{E} \sim 1 \mathrm{kV} / \mathrm{m}$, which in turn leads to $\xi \sim 3 \mathrm{meV}$. In this manner, our perturbative results would allow for an analytical treatment of future experimental extensions of the work described in Ref. [7], in case they would include a quantizing magnetic field in their study. It would also allow for larger values of the radiation field intensity with photoinduced features in the Landau level structure of single-layer graphene as it is described in the following.

For this purpose we transform the Hamiltonian (20) as $H=e^{(\lambda / 2) I_{-}} H_{F} e^{-(\lambda / 2) I_{-}}$, where we have introduced the antiHermitian operator $I_{-}=a^{\dagger} \sigma_{-}-a \sigma_{+}$. Evaluating up to first order we get

$$
H \approx H_{F}+\frac{\lambda}{2}\left[I_{-}, H_{F}\right] .
$$

Evaluation of the commutator gives (in the Appendix we summarize the explicit derivations)

$$
\left[I_{-}, H_{F}\right]=-\hbar \omega_{c}\left[2 N_{a}+\lambda\left(a^{\dagger}+a\right)\right] \sigma_{z},
$$

and defining the shifted operator $b=a+\lambda$ one gets, to leading order in $\lambda$, the effective Hamiltonian

$$
H=\hbar \omega_{c}\left(b^{\dagger} \sigma_{-}+b \sigma_{+}\right)-\delta \hbar \omega N_{b}-\xi N_{b} \sigma_{z},
$$

where we have introduced the shifted number operator

$$
N_{b}=b^{\dagger} b+\frac{\mathbb{1}+\sigma_{z}}{2} .
$$

In Eq. (23) we have neglected the additive higher order terms

$$
\Delta V=\hbar \omega \lambda\left(b^{\dagger}+b\right)-\lambda^{2} \hbar \omega,
$$

which can be dealt with by higher order perturbation theory. Thus, the approximate quasienergies are found to be given as

$$
\epsilon_{m}^{s}=s \hbar \omega_{c} \sqrt{m} \sqrt{1+m \lambda^{2}}, \quad \bmod \hbar \omega,
$$

which can be rewritten as

$$
\epsilon_{m}^{s}=s \sqrt{m\left(\hbar \omega_{c}\right)^{2}+(m \xi)^{2}}, \quad \bmod \hbar \omega .
$$

Then, to this order of approximation, all quasienergies corresponding to the $m \neq 0 \mathrm{LL}$ are shifted, whereas the $m=0$ remains insensitive to the radiation field. This shift of all but the $m=0$ quasienergy LL spectrum agrees with the result reported in Ref. [19] for bilayer graphene. Yet, a more detailed derivation by means of second order perturbation theory shows that there is a small $O\left(\lambda^{4}\right)$ energy correction due to first nondiagonal terms in Eq. (24) which couple all adjacent LL. This higher order corrections could be important at low quantizing magnetic fields for which the condition $\lambda=\xi / \hbar \omega_{c} \approx 1$ could be satisfied.

Let us now analyze some physical consequences of the radiation field on the Landau level structure of monolayer graphene with focus on the interplay among the quantizing magnetic field and the light-matter interaction. To begin with, we notice that the quasienergies are defined up to multiples of $\omega$; therefore, a better physical characterization of the energy spectrum for the driven system is provided by the mean energies [17]

$$
\bar{\epsilon}_{m}^{s}=\epsilon_{m}^{s}-\omega \frac{\partial \epsilon_{m}^{s}}{\partial \omega},
$$

which are invariant under $\epsilon_{m}^{s} \rightarrow \epsilon_{m}^{s}+l \hbar \omega$, for $l$ being an integer. Doing the explicit calculation the mean energies are found to be given by the expression

$$
\bar{\epsilon}_{m}^{s}=s\left(\frac{m\left(\hbar \omega_{c}\right)^{2}+2 m^{2} \xi^{2}}{\sqrt{m\left(\hbar \omega_{c}\right)^{2}+m^{2} \xi^{2}}}\right),
$$

where we remember the definition of the effective coupling to the radiation field as $\xi=e v_{F} \mathcal{E} / \omega$.

As can be seen in Fig. 1, these mean energies are plotted as a function of the quantizing magnetic field $B$, for different values of the Landau level index changing the effective coupling $\xi$. We notice that, at intermediate light-coupling strength, the energy resolution of these levels becomes much better and could experimentally be tested for not so large quantizing magnetic fields $B$. Moreover, we find that to this order of approximation the LL become gapped, with the striking feature that the photoinduced gap is level dependent. These gap openings appear except for the $m=0$ level which, as discussed before, remains insensitive to the radiation field. 


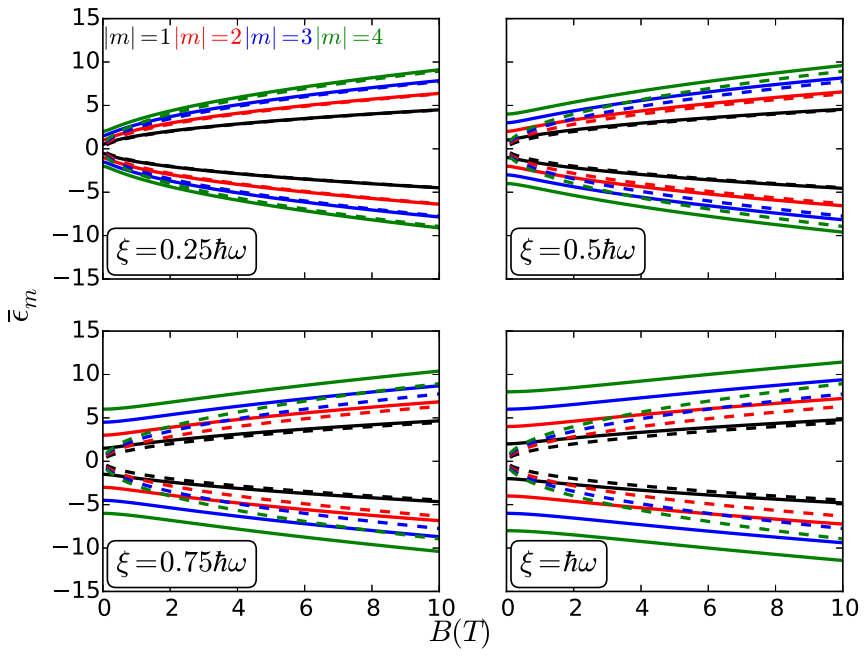

FIG. 1. (Color online) Approximate mean energies for the driven scenario (continuous lines) as a function of the quantizing magnetic field $B$. The dotted lines represent the undriven spectra for the corresponding LL. We notice a level-dependent energy gap that leads to nontrivial behavior of physical quantities, as discussed below (see main text).

Below we will deal with the photoinduced dynamical features and therefore we give the corresponding normalized Floquet eigenstates for $m \neq 0$,

$$
\left|\psi_{m}^{s}\right\rangle=\left(\begin{array}{c}
s f_{m}^{-s}|m-1\rangle \\
f_{m}^{s}|m\rangle
\end{array}\right),
$$

where we have defined the coefficients

$$
f_{m}^{s}=\sqrt{\frac{\epsilon_{m}+s m \xi}{2 \epsilon_{m}}}
$$

with $\epsilon_{m}=\left|\epsilon_{m}^{s}\right|$. In addition, the zero energy eigenstate $(m=0)$ is still given by $\left|\varphi_{0}\right\rangle$, i.e.,

$$
\left|\psi_{0}\right\rangle=\left(\begin{array}{c}
0 \\
|0\rangle
\end{array}\right)
$$

\section{PSEUDOSPIN AND AUTOCORRELATION FUNCTION DYNAMICS}

Now that we have found the approximate Floquet eigenstates and quasienergies, we explore other dynamical features of the driven LL configuration by evaluating the mean values of the pseudospin polarization operator and its relation to the autocorrelation function dynamics [20].

For this purpose, let us first assume that the system is initially prepared in an eigenstate of the Hamiltonian $H_{0}$,

$$
|\Psi(0)\rangle=\left|\varphi_{m}^{s}\right\rangle,
$$

with $m \neq 0$. In the Floquet basis (29), the initial state is written as

$$
\left|\varphi_{m}^{s}\right\rangle=\sum_{s^{\prime}= \pm s} D_{m}^{s s^{\prime}}\left|\psi_{m}^{s^{\prime}}\right\rangle
$$

where the expansion coefficients are given by

$$
D_{m}^{s s^{\prime}}=\frac{1}{\sqrt{2}}\left(f_{m}^{s^{\prime}}+s s^{\prime} f_{m}^{-s^{\prime}}\right) .
$$

Taking into account that the $\left|\psi_{m}^{ \pm}\right\rangle$states are degenerate eigenstates of $N_{b}$, the unitary operator $e^{-i N_{b} \omega t}$ would just contribute a phase $e^{-i \omega m t}$. Using the Hamiltonian (23) the evolved state can be written as

$$
|\Psi(t)\rangle=\sum_{s^{\prime}= \pm s} D_{m}^{s s^{\prime}}(t) e^{-i s^{\prime} \epsilon_{m} t / \hbar}\left|\psi_{m}^{s^{\prime}}\right\rangle,
$$

where we have introduced the time-dependent coefficients $D_{m}^{s s^{\prime}}(t)=D_{m}^{s s^{\prime}} e^{-i \omega m t}$. Let us then consider the dynamics for the autocorrelation function and pseudospin polarization $\sigma_{z}(t)$ operators from which we can respectively infer the feasibility of manipulating the polarization state of the sample and the dipole moment radiation emitted by the driven sample. It is important to remark at this point that studying the out-of-plane pseudospin polarization is a means of detecting the angular momentum exchange between the Dirac fermions in graphene and the circularly polarized radiation field, as can be inferred from the discussion in the recent literature about the role of $\sigma_{z}$ in describing the total angular momentum content of the system [14].

With these ideas in mind, we first begin by evaluating the pseudospin polarization

$$
\sigma_{z}(t, \xi)=\left\langle\Psi(t)\left|\sigma_{z}\right| \Psi(t)\right\rangle .
$$

For the chosen initial state we find $\sigma_{z}(t, 0)=0$. When expression (36) is evaluated we find after some algebraic manipulations

$$
\sigma_{z}(t, \xi)=\frac{2 s \sqrt{m^{3}} \xi \hbar \omega_{c}}{\epsilon_{m}^{2}} \sin ^{2} \epsilon_{m} t / \hbar .
$$

We note that for vanishing values of the coupling to the radiation field $\xi \rightarrow 0$ one has $\sigma_{z}(t, 0)=0$. Therefore, once the electromagnetic field is present the pseudospin oscillations are a manifestation of the angular momentum exchange among the radiation field and the charge carriers in graphene [14].

As discussed in the case of the quasienergies, we could better quantify the effects of the driving field by evaluating the average

$$
\left\langle\sigma_{z}\right\rangle=\frac{1}{T} \int_{0}^{T} d t \sigma_{z}(t, \xi),
$$

with the period of the radiation field given as $T=2 \pi / \omega$. Then we get the expression

$$
\left\langle\sigma_{z}\right\rangle=\frac{s \sqrt{m^{3}} \xi \hbar \omega_{c}}{\epsilon_{m}^{2}}\left(1-\operatorname{sinc} 2 \epsilon_{m} T / \hbar\right),
$$

with $\operatorname{sinc} x=\sin x / x$.

In Fig. 2 we plot the behavior of $\left\langle\sigma_{z}\right\rangle$ as a function of the coupling strength $\xi$. At small $\xi=0.25 \hbar \omega$ we see that the closer the state is to the $m=0 \mathrm{LL}$ the role of the radiation field in modifying its pseudospin polarization is less relevant and this is correlated to the fact that, within this regime, the gap openings seen in the mean quasienergy spectrum are not so noticeable at different values of the $m$ LL index. In addition, at large values of the quantizing field, $B=10 \mathrm{~T}$, the value of the 

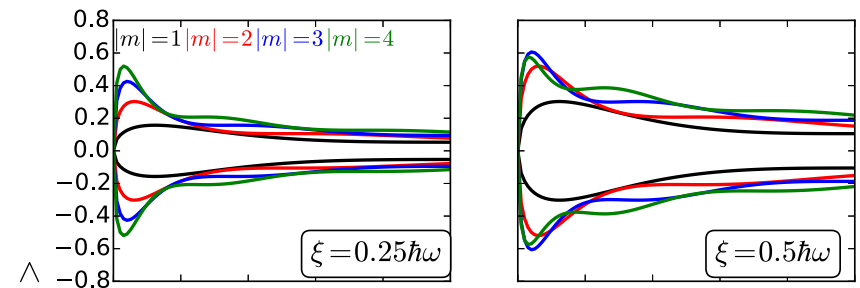

$b^{N}$

0.8
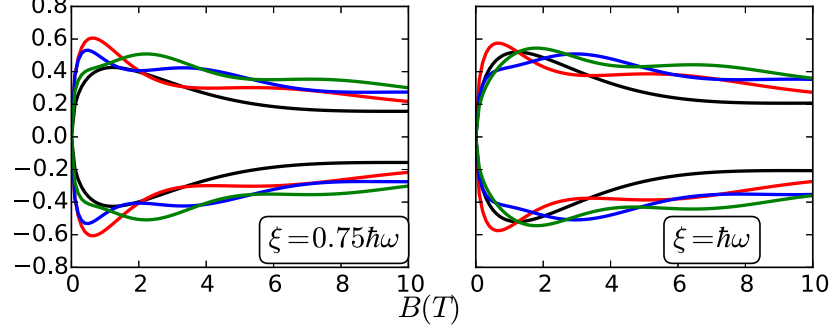

FIG. 2. (Color online) Approximate mean pseudospin polarization $\left\langle\sigma_{z}\right\rangle$ for the driven scenario as given by Eq. (39), plotted as a function of the quantizing magnetic field $B$. We have selected the first four LL $m=1, \ldots, 4$, at four values of the light-matter coupling strength. The lower panels show that at large magnetic fields $(B=10 \mathrm{~T})$ both the $m=3$ and $m=4 \mathrm{LL}$ the pseudospin polarization still remains degenerate. Hence higher order perturbation terms should be necessary in order to lift this degeneracy.

pseudospin polarization is almost the same at each value of the $m$ LL index. However, we see in the second upper panel that already at intermediate values of the relative coupling strength $\xi=0.5 \hbar \omega$ that at large values of the quantizing field $B=10 \mathrm{~T}$ one can discern among the different LL polarization values which serve to separate each level contribution to this quantity dynamical behavior. In the lower two panels we show that this physical picture becomes more obvious at larger values of $\xi$, with perfect separation for the contribution from the $m=1$ and $m=2$ levels. Yet, the corresponding $m=3$ and $m=4$ contributions to the pseudospin polarization remain degenerate at large $B$ values. One would expect that this accidental crossing seen as a degeneracy would be removed by including higher order perturbative contributions, since then the coupling among nearby levels would lead to additional splittings in the quasienergy spectrum that would in turn also lift this accidental pseudospin degeneracy.

In order to discuss more general dynamical features, one should consider a superposition state. However, in order to do so we must take into account the feasibility of experimentally realizing such a superposition state. A paradigmatic case of such interesting superposition states is given by the coherent state which are minimal uncertainty wave packets relevant for studying the classical states of the radiation field in the sense of being a classical counterpart of the quantum harmonic oscillator. Since, as in the quantum harmonic oscillator, the Landau levels in graphene are eigenstates of the number operator, one should expect that these coherent state superpositions would be interesting. Indeed, there has been recently some proposals to analyze the dynamics of coherent electronic states in graphene nanomechanical resonators [21]. By taking advantage of the intrinsic nonlinear nature of flexural modes in graphene, the authors of Ref. [21] show that catlike
[22] states can be generated. We follow a different physical approach. Instead of resorting to nonlinearities of flexural modes we invoke the light-matter coupling as a mechanism for studying the evolution of an initially prepared coherent superposition state built from the Landau level eigenstates described in Sec. II and evaluate the induced pseudospin polarization effects in order to contrast to the results shown in Fig. 2.

Formally speaking, the coherent state $|\alpha\rangle$ is defined by means of the eigenvalue equation

$$
A|\alpha\rangle=\alpha|\alpha\rangle,
$$

where $A=a \mathbb{1}$. Using the expansion

$$
|\alpha\rangle=c_{0}\left|\varphi_{0}\right\rangle+\sum_{s n}^{-} c_{n}^{s}\left|\varphi_{n}^{s}\right\rangle,
$$

with $\sum_{s n}$ representing a summation for all $n \neq 0$. Assuming, without loss of generality, the symmetric scenario $c_{n}^{-s}=c_{n}^{s}$, one finds that the coherent state is given as

$$
|\alpha\rangle=e^{-\frac{|\alpha|^{2}}{2}}\left(\left|\varphi_{0}\right\rangle+\frac{1}{\sqrt{2}} \sum_{s n}^{-} \frac{\alpha^{n}}{\sqrt{n !}}\left|\varphi_{n}^{s}\right\rangle\right),
$$

which can be shown to be normalized. If we now evaluate the mean value of the Hermitian operator $A^{\dagger} A$ in the coherent state and use the definition given in Eq. (40), we get $\left\langle\alpha\left|A^{\dagger} A\right| \alpha\right\rangle=$ $|\alpha|^{2}$. In addition, if we use the coherent state to evaluate the average of the number operator $N_{a}$ defined in Eq. (16), it is not difficult to show that we also get $\left\langle\alpha\left|N_{a}\right| \alpha\right\rangle=|\alpha|^{2}$. In order to do so, we just need to use the result $\left\langle\alpha\left|\sigma_{z}\right| \alpha\right\rangle=-1$. Therefore, the coherent state parameter amplitude $|\alpha|$ is a measure of the mean number of Landau levels that are excited and corresponds to the mean photon number in the context of quantum optics. Thus, we will use it as a control parameter to discuss the properties of the physical quantities as follows.

In this case, we find for the pseudospin polarization

$$
\left\langle\alpha\left|\sigma_{z}(t)\right| \alpha\right\rangle=-e^{-|\alpha|^{2}} \sum_{n} \frac{|\alpha|^{2 n}}{n !}\left(\frac{\left(\hbar \omega_{c}\right)^{2} \cos 2 \epsilon_{n} t / \hbar+n \xi^{2}}{\left(\hbar \omega_{c}\right)^{2}+n \xi^{2}}\right) .
$$

This is plotted in Fig. 3 for $\xi=0.25 \hbar \omega$ and four representative values of $|\alpha|$. In this figure we notice that for small values of $|\alpha|$ the dynamics of the pseudospin polarization resembles the pattern for Rabi oscillations since the main contributions would arise for the interference among the zero and first LL. Yet, no polarization inversion can be achieved within this regime.

However, once $|\alpha|=1$ the contribution from other LL states becomes increasingly important to the interference pattern and the former Rabi oscillations become distorted. Moreover, at this value of $|\alpha|$, one can achieve the polarization inversion for large enough values of time in the long-term evolution. In the lower panels of Fig. 4 we find that for larger values $(|\alpha|=4$ and $|\alpha|=8)$ we get a beating pattern showing a dynamical localization effect that is directly related to a collective behavior of the driven charge carries in graphene. We would like to remark that the problem of the population inversion has already been studied a long time ago by Eberly et al. [23]. Their model corresponds to a two-level system 

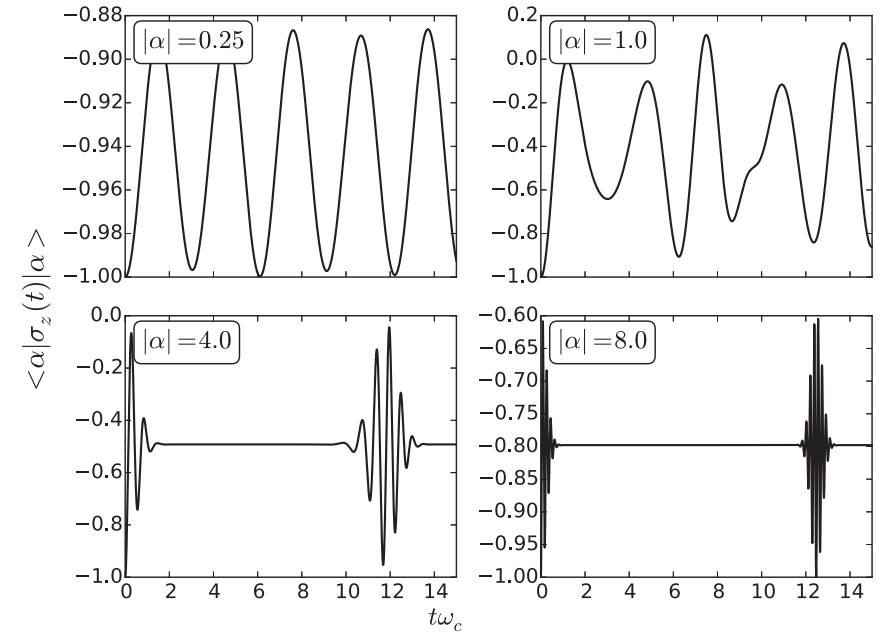

FIG. 3. Time dependence of the pseudospin polarization as given in Eq. (43). The panels show the dynamical behavior at four characteristic values $|\alpha|$. For the numerical evaluation of the series we have truncated at $n=N=100$. The upper panels show the coherence among the lowest LL, whereas the lower panel show localization effects approaching the classical behavior, which corresponds to large values of $|\alpha|$. See the discussion in the main text.

couple to a single mode radiation field (Jaynes-Cummings Hamiltonian). In our approach this in turn is given by the static graphene Hamiltonian for Landau levels written in Eq. (2). A larger value of the coherent state parameter $|\alpha|$ would imply larger mean Landau level occupation, and thus the second term in the numerator of the pseudospin polarization given in expression (43) would have a larger influence in the pseudospin inversion since larger $n$ Landau levels would be occupied which will contribute a higher weight in the pseudospin polarization [see numerator in Eq. (43)]. Moreover,

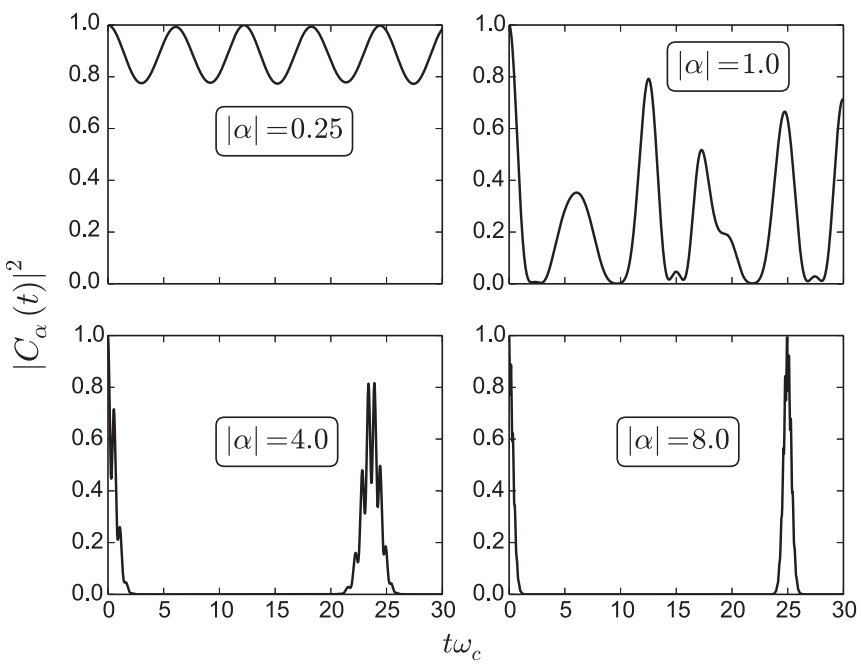

FIG. 4. Time dependence of the autocorrelation function as given in Eq. (44) truncated at $n=N=100$. For large $|\alpha|=4$ and $|\alpha|=8$, it is the cyclotron frequency $\omega_{c}$ instead of the driving frequency $\omega$ which determines the time scale for the revival times. Comparing to Fig. 3, it is apparent that information on the pseudospin dynamics can be indirectly inferred from the autocorrelation function dynamics. See discussion in the main text.

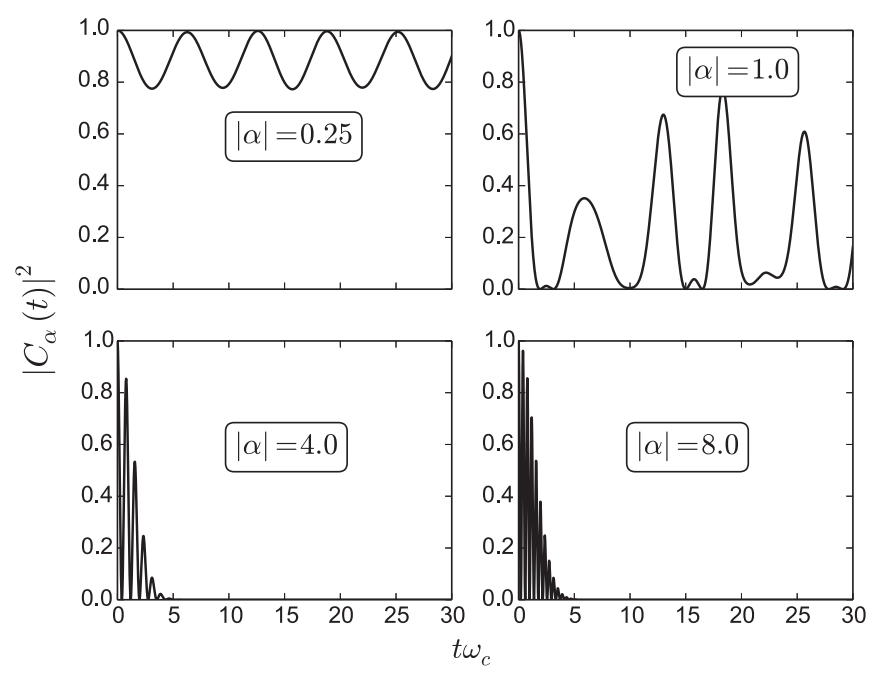

FIG. 5. Time dependence of the autocorrelation function as given in Eq. (44) truncated at $n=N=100$ in absence of radiation field $\xi=0$. In the upper panels we see that at small values of $|\alpha|=0.25$ and $|\alpha|=1$, the static and driven autocorrelation function are qualitatively similar. Yet, the photoinduced quantum revivals at larger times are not seen in the two lower panels as compared to Fig. 4.

since the effective light-matter coupling strength $\xi$ also affects the phase, as given by the cosine term in Eq. (43) of the polarization, this interesting interplay forbids the inversion which is also a signature of the localization and beating effects shown in Figs. 3(c) and 3(d) and our results extend those found in the context of Ref. [23].

This physical picture for the coherent state dynamics can be complemented by studying the autocorrelation function $C_{\alpha}(t)=\langle\alpha \mid \Psi(t)\rangle$, which is found to be given as

$$
C_{\alpha}(t)=e^{-|\alpha|^{2}} \sum_{n} \frac{|\alpha|^{2 n}}{n !}\left(\cos \epsilon_{n} t / \hbar+i \frac{n \xi}{\epsilon_{n}} \sin \epsilon_{n} t / \hbar\right) .
$$

The autocorrelation function provides additional physical information of the system since its Fourier transform is related to the local density of states [20]. Its time evolution is plotted in Fig. 4 choosing again an effective coupling strength value of $\xi=0.25 \hbar \omega$ and the same values of $\alpha$ as in Fig. 3 showing the dynamical behavior of the pseudospin polarization. Comparing Figs. 3 and 4 we see that partial revivals for the autocorrelation function are correlated to the beating or localization behavior of the pseudospin polarization. Therefore, one could indirectly gain information on the pseudospin dynamics by measuring the time revivals [20] which means detecting those times for which the wave packet reconstructs itself. In order to show explicitly the role of the driving field we have plotted in Fig. 5 the static autocorrelation function. Comparing the lower right panels in Figs. 4 and 5, we find that the radiation field induces additional revivals in accordance to the beating pattern in the pseudospin oscillation, as seen in Fig. 3.

\section{DISCUSSION}

We have shown that the radiation field leads to a quasienergy spectrum with a level dependent gap, except for the $m=0 \mathrm{LL}$ 
which, to leading order, remains insensitive to the radiation field effects. In addition, we have found that a finite outof-plane pseudospin polarization value can arise for initial states that possess either a finite or vanishing initial value of $\sigma_{z}$. As we saw at the beginning of Sec. II, in presence of the radiation field, the total out-of-plane angular momentum component $j_{z}$ of the electrons is no longer a constant of motion. Therefore, the pseudospin oscillations are due to the angular momentum exchange among the driving field and the charge carriers in single layer graphene. For the coherent state a beating pattern emerges in the pseudospin polarization. In spite of the fact that the interaction is dictated by a periodic Hamiltonian we find that the dominant or characteristic time scale for this collective behavior is the cyclotron frequency. We could expect this behavior of the wave packet at large values of $|\alpha|$ as a measure of the classical behavior associated with the cyclotron problem but now realized with Dirac fermions in the LL quantized regime as was discussed by one of the authors in Ref. [24]. We must remark that in order to be able to detect the reported effects an ensemble of coherent states should be prepared each time, because the measurement process destroys the state. It should also be remembered that the coherent state parameter $|\alpha|$ is determined by choosing appropriate values for the mean values of position and momentum in accordance to the following prescription:

$$
\begin{aligned}
\langle\alpha|x| \alpha\rangle & =\ell_{B} \operatorname{Re}(\alpha), \\
\left\langle\alpha\left|p_{x}\right| \alpha\right\rangle & =\frac{1}{\ell_{B}} \operatorname{Im}(\alpha) .
\end{aligned}
$$

Another point to be highlighted is that for standard twodimensional electron gases the coherent state built from the Landau levels would remain coherent, i.e., it will evolve in time in such a manner that it would just oscillate in time around the prescribed mean values given in Eqs. (45) and (46). More precisely, its Wigner function representation in phase space will oscillate in time without deformation. This is known to be a consequence of the fact that the dependence with the $n$ quantum number is linear. Yet, in graphene we have a $\sqrt{n}$ and this in turn prevents the coherent state to evolve in such a coherent manner.

Therefore, the spreading of the wave packet and the corresponding appearance of the additional revival times at shorter time values is a direct consequence of the driving field that even at low coupling can induce interesting dynamical behavior as shown in Figs. 3 and 4. We supported this last statement by evaluating the static $(\xi=0)$ autocorrelation function as shown in Fig. 5 and found that the second packet reconstruction (see lower panel for $|\alpha|=8$ in Fig. 4) around $t \omega_{c}=24$ is absent in the static regime.

We would also like to comment that graphene subject to electromagnetic radiation without quantizing magnetic field has been discussed in several papers [5]. It has been shown that for zero momentum $(k=0)$, the dynamical equation is exactly solvable and leads to a photoinduced (zero quantizing magnetic field) mass term that has opposite signs at the two valleys. Yet, at finite momentum the dynamics is no longer exactly solvable and one has to resort to numerical analysis by means of an infinite expansion in Fourier modes. This is why we consider our results to be a valuable tool in analytically describing the photoinduced pseudospin effects in the Landau level structure of monolayer graphene.

We now briefly mention that our values of quantizing magnetic fields are within experimentally accessible orders. For instance, in the pioneering paper by Novoselov et al. in Ref. [25], the authors used a value of quantizing magnetic field $B=14$ T to study the quantum Hall effect in graphene. Moreover, in a following classical paper, their results were extended up to values of $B=29 \mathrm{~T}$ and even $B=45 \mathrm{~T}$ for the quantizing magnetic field [26]. Here it is shown that at these values of magnetic field, the quantum Hall effect in graphene could be observed at room temperature that constitutes a breakthrough in the physics of quantum Hall phases.

\section{CONCLUSIONS}

We have analyzed the dynamical modulation of physical quantities for Dirac fermions within the Landau level quantized regime of single layer graphene subject to intense circularly polarized Terahertz radiation. To the best of our knowledge, this is the first analysis of the photoinduced manipulation of the LL structure in single-layer graphene subject to a continuously applied intense laser field instead of a pulsed one. By means of a perturbative analytical treatment we found very interesting physical features such as a nontrivial level dependent dynamically induced gap structure. Due to the angular momentum exchange among the radiation field and the charge carriers, it also leads to modulation of the oscillations in the dynamics of the out-of-plane pseudospin polarization, even for superposition states with an initially vanishing pseudospin value. We also found that localization effects in the time evolution of the pseudospin polarization can be kept track of by measuring the revival times of a wave packet initially prepared as a coherent state. The reported photoinduced gap modulation and pseudospin oscillations could be detected through the re-emitted dipolar radiation from the oscillating charge carriers as it was proposed in Ref. [13].

We would also like to mention that for values of $\lambda \gg 1$, one enters the so called ultrastrong coupling limit in the context of cavity quantum electrodynamics experiments. Within this parameter regime, it has been experimentally shown in Ref. [27] that explicit anticrossings appear in the energy spectrum. This in turn corresponds to a breakdown of the Jaynes-Cummings approximation that describes resonant processes in the two-level problem coupled to a single mode radiation field. Our exact Floquet Hamiltonian given in Eq. (20) allows us to explore this ultrastrong coupling regime beyond the perturbative results presented and could be the subject of future work where one would expect nonlinear effects to be relevant in the graphene physics. For instance, as we already argued in the Discussion section, one could expect that the accidental degeneracies seen in the panels of Fig. 2, at large values of the quantizing magnetic field, would be lifted by a stronger coupling to the radiation field.

Moreover, we would like to remark that although we perform our analysis for neutral undoped graphene samples, in the graphene literature the role of doping has been given special attention since it might modify the effective Fermi velocity of Dirac fermions [28,29]. The first work [28] shows that the energy spectrum near the charge neutrality point is 
nonlinear and no gap is found at energies even as close to the Dirac point as $0.1 \mathrm{meV}$. On the other hand, the authors of Ref. [29] show that electron interactions leave the graphene energy dispersion linear as a function of excitation energy even for energies within $\pm 200 \mathrm{meV}$ of the Fermi energy. Yet, it has recently been shown that doping effects might also lead to a band gap opening in the graphene spectrum (see Ref. [30], and references therein). In particular, the authors of the work [30] have reviewed the role of heteroatom doping. Another paper addresses the tunability of the band gap energy in single-layer graphene due to manganese oxide nanoparticles by means of an electrochemical method [31]. This work reports a maximum value for the induced energy band gap of $0.256 \mathrm{eV}$. In addition, another experimental paper [32] deals with the band gap engineering in monolayer graphene via boron-nitrogen (BN) doping. They argue that a significant band gap as high as $600 \mathrm{meV}$ is observed for low $\mathrm{BN}$ concentrations and is attributed to the opening of the $\pi-\pi^{*}$ band gap of graphene due to isoelectronic $\mathrm{BN}$ doping. Although they do not discuss the quantized LL regime, we could incorporate these doping effects in our model by including a phenomenological diagonal term in the Landau level Hamiltonian given in Eqs. (2) and (3). This mass term would in turn be proportional to $\sigma_{z}$ and the valley index $\eta$, as required by time reversal invariance. Thus, one would expect an interesting interplay between the photoinduced gap openings and the static band gap determined by doping effects. Yet, we considered undoped graphene in order to highlight the photoinduced LL dependent band gap discussed above.

Concerning the actual experimental observation of our predicted results, we consider that these could motivate the exploration of larger intensities for the radiation field as given in the experimental setup in Ref. [7] when their model enters the Landau level regime. In this sense, we consider that our work would contribute to explore a physical scenario within realistic parameter values to discuss the pseudospin physics in graphene, also taking into account the role of Zitterbewegung as it is discussed in Ref. [13], extending their results to initially prepared coherent states when the radiation field is a monochromatic continuous laser field instead of a pulsed laser.

\section{ACKNOWLEDGMENTS}

This work has been supported by Deutsche Forschungsgemeinschaft via GRK 1570 and Yachay Tech via the project "Non dissipative transport in honeycomb lattice materials:
Interplay of spin-orbit interaction, radiation fields and superconductivity." A.L. thanks the Universite de Lorraine for partial financial support through research visits. B.B. thanks Yachay Tech for financial support for research visits.

\section{APPENDIX: PERTURBATIVE CALCULATION OF EFFECTIVE HAMILTONIAN FOR SINGLE LAYER GRAPHENE}

In order to get the effective Hamiltonian for single layer graphene we need to evaluate the following expression:

$$
H=e^{\lambda / 2 I_{-}} H_{F} e^{-\lambda / 2 I_{-}},
$$

with the anti-Hermitian operator

$$
I_{-}=\hat{a}^{\dagger} \sigma_{-}-\hat{a} \sigma_{+} .
$$

Using the Baker-Campbell-Hausdorff formula we have

$$
H=H_{F}+\frac{\lambda}{2}\left[I_{-}, H_{F}\right]+\frac{1}{2 !}\left(\frac{\lambda}{2}\right)^{2}\left[I_{-},\left[I_{-}, H_{F}\right]\right]+\cdots .
$$

The first commutator is worked out explicitly:

$$
\left[I_{-}, H_{F}\right]=\left[\hat{a}^{\dagger} \sigma_{-}-\hat{a} \sigma_{+}, H_{F}\right] .
$$

Since $H_{F}=\omega_{c} I_{+}-\omega \hat{N}_{a}+\xi \sigma_{x}$ and $\left[I_{-}, \hat{N}_{a}\right]=0$, with $I_{+}=$ $\hat{a}^{\dagger} \sigma_{-}+\hat{a} \sigma_{+}$, we only need to evaluate two commutators. The first one gives

$$
\begin{aligned}
{\left[I_{-}, I_{+}\right] } & =\left[\hat{a}^{\dagger} \sigma_{-}-\hat{a} \sigma_{+}, \hat{a}^{\dagger} \sigma_{-}+\hat{a} \sigma_{+}\right]=-2\left[\hat{a} \sigma_{+}, \hat{a}^{\dagger} \sigma_{-}\right] \\
& =-2\left(\hat{a}\left[\sigma_{+}, \hat{a}^{\dagger} \sigma_{-}\right]+\left[\hat{a}, \hat{a}^{\dagger} \sigma_{-}\right] \sigma_{+}\right) \\
& =-2\left(\hat{a} \hat{a}^{\dagger}\left[\sigma_{+}, \sigma_{-}\right]+\left[\hat{a}, \hat{a}^{\dagger}\right] \sigma_{-} \sigma_{+}\right) \\
& =-2\left(\hat{a} \hat{a}^{\dagger} \sigma_{z}+\left(\mathbb{1}-\sigma_{z}\right) / 2\right) \\
& =-2\left(\hat{a}^{\dagger} \hat{a} \sigma_{z}+\left(\mathbb{1}+\sigma_{z}\right) / 2\right) \\
& =-2\left(\hat{a}^{\dagger} \hat{a}+\left(\mathbb{1}+\sigma_{z}\right) / 2\right) \sigma_{z}=-2 \hat{N}_{a} \sigma_{z},
\end{aligned}
$$

whereas the second one follows as

$$
\begin{aligned}
{\left[I_{-}, \sigma_{x}\right] } & =\left[\hat{a}^{\dagger} \sigma_{-}-\hat{a} \sigma_{+}, \sigma_{-}+\sigma_{+}\right] \\
& =-\hat{a}\left[\sigma_{+}, \sigma_{-}\right]+\hat{a}^{\dagger}\left[\sigma_{-}, \sigma_{+}\right] \\
& =-\left(\hat{a}+\hat{a}^{\dagger}\right) \sigma_{z} .
\end{aligned}
$$

Upon substitution of these first-order corrections and introduction of the shifted harmonic oscillator operators we get the effective Hamiltonian given in Eq. (23).
[1] K. S. Novoselov, A. K. Geim, S. V. Morozov, D. Jiang, Y. Zhang, S. V. Dubonos, I. V. Grigorieva, and A. A. Firsov, Science 306, 666 (2004).

[2] A. K. Geim and K. S. Novoselov, Nat. Mater. 6, 183 (2007).

[3] A. H. Castro Neto, F. Guinea, N. M. R. Peres, K. S. Novoselov, and A. K. Geim, Rev. Mod. Phys. 81, 109 (2009).

[4] T. Oka and H. Aoki, Phys. Rev. B 79, 081406(R) (2009).

[5] H. L. Calvo, H. M. Pastawski, S. Roche, and L. E. F. Foa Torres, Appl. Phys. Lett. 98, 232103 (2011).

[6] Y. Zhou and M. W. Wu, Phys. Rev. B 83, 245436 (2011).
[7] J. Karch, P. Olbrich, M. Schmalzbauer, C. Zoth, C. Brinsteiner, M. Fehrenbacher, U. Wurstbauer, M. M. Glazov, S. A. Tarasenko, E. L. Ivchenko, D. Weiss, J. Eroms, R. Yakimova, S. Lara-Avila, S. Kubatkin, and S. D. Ganichev, Phys. Rev. Lett. 105, 227402 (2010).

[8] C. L. Kane and E. J. Mele, Phys. Rev. Lett. 95, 226801 (2005).

[9] T. Kitagawa, E. Berg, M. Rudner, and E. Demler, Phys. Rev. B 82, 235114 (2010).

[10] T. Kitagawa, T. Oka, A. Brataas, L. Fu, and E. Demler, Phys. Rev. B 84, 235108 (2011). 
[11] Z. Gu, H. A. Fertig, D. P. Arovas, and A. Auerbach, Phys. Rev. Lett. 107, 216601 (2011).

[12] B. Dóra, K. Ziegler, P. Thalmeier, and M. Nakamura, Phys. Rev. Lett. 102, 036803 (2009).

[13] T. M. Rusin and W. Zawadzki, Phys. Rev. B 80, 045416 (2009).

[14] M. Mecklenburg and B. C. Regan, Phys. Rev. Lett. 106, 116803 (2011).

[15] D. Yudin, O. Eriksson, and M. I. Katsnelson, Phys. Rev. B 91, 075419 (2015).

[16] J. Cayssol, B. Dóra, F. Simon, and R. Moessner, Phys. Status Solidi RRL 7, 101 (2013).

[17] M. Grifoni and P. Hänggi, Phys. Rep. 304, 229 (1998).

[18] Q.-g. Lin, J. Phys. A: Math. Gen. 34, 1903 (2001).

[19] D. S. L. Abergel and T. Chakraborty, Nanotechnology 22, 015203 (2011).

[20] R. W. Robinett, Phys. Rep. 392, 1 (2004); V. Krueckl and T. Kramer, New J. Phys. 11, 093010 (2009); E. Romera and F. de los Santos, Phys. Rev. B 80, 165416 (2009); E. Romera, Phys. Rev. A 84, 052102 (2011).

[21] A. Voje, J. M. Kinaret, and A. Isacsson, Phys. Rev. B 85, 205415 (2012).

[22] D. Leibfried, E. Knill, S. Seidelin, J. Britton, R. B. Blakestad, J. Chiaverini, D. Hume, W. M. Itano, J. D. Jost, C. Langer, R. Ozeri, R. Reichle, and D. J. Wineland, Nature 438, 639 (2005).

[23] J. H. Eberly, N. B. Narozhny, and J. J. Sanchez-Mondragon, Phys. Rev. Lett. 44, 1323 (1980).
[24] J. Schliemann, New J. Phys. 10, 043024 (2008).

[25] K. S. Novoselov, A. K. Geim, S. V. Morozov, D. Jiang, M. I. Katsnelson, I. V. Grigorieva, S. V. Dubonos, and A. A. Firsov, Nature (London) 438, 197 (2005).

[26] K. S. Novoselov, Z. Jiang, Y. Zhang, S. V. Morozov, H. L. Stormer, U. Zeitler, J. C. Maan, G. S. Boebinger, P. Kim, and A. K. Geim, Science 315, 1379 (2007).

[27] T. Niemczyk, F. Deppe, H. Huebl, E. P. Menzel, F. Hocke, M. J. Schwarz, J. J. Garcia-Ripoll, D. Zueco, T. Hümmer, E. Solano, A. Marx, and R. Gross, Nat. Phys. 6, 772 (2010).

[28] D. C. Elias, R. V. Gorbachev, A. S. Mayorov, S. V. Morozov, A. A. Zhukov, P. Blake, L. A. Ponomarenko, I. V. Grigorieva, K. S. Novoselov, F. Guinea, and A. K. Geim, Nat. Phys. 7, 701 (2011).

[29] J. Chae, S. Jung, A. F. Young, C. R. Dean, L. Wang, Y. Gao, K. Watanabe, T. Taniguchi, J. Hone, K. L. Shepard, P. Kim, N. B. Zhitenev, and J. A. Stroscio, Phys. Rev. Lett. 109, 116802 (2012).

[30] X. Wang, G. Sun, P. Routh, D.-H. Kim, W. Huang, and P. Chen, Chem. Soc. Rev. 43, 7067 (2014).

[31] C. S. Park, Y. Zhao, J.-H. Lee, D. Whang, Y. Shon, Y.-H. Song, and C. J. Lee, Appl. Phys. Lett. 102, 032106 (2013).

[32] C.-K. Chang, S. Kataria, C.-C. Kuo, A. Ganguly, B.-Y. Wang, J.-Y. Hwang, K.-J. Huang, W.-H. Yang, S.-Bo Wang, C.-H. Chuang, M. Chen, C.-I. Huang, W.-F. Pong, K.-J. Song, S.-J. Chang, J.-H. Guo, Y. Tai, M. Tsujimoto, S. Isoda, C.-W. Chen, L.-C. Chen, and K.-H. Chen, ACS Nano 7, 1333 (2013). 